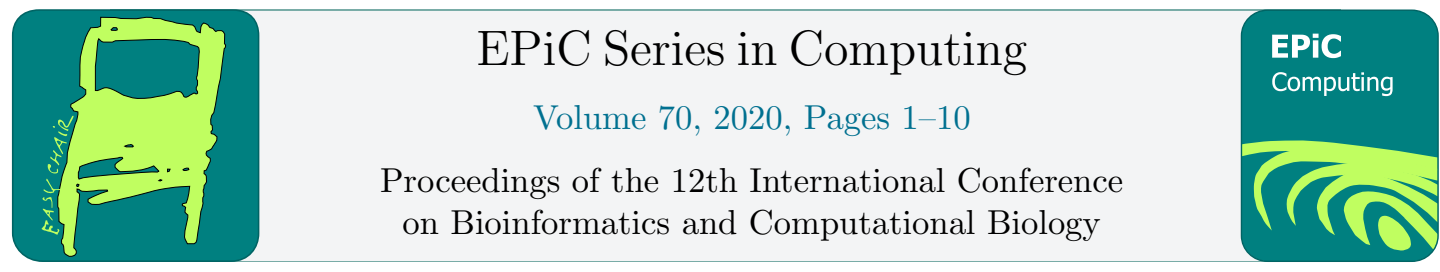

\title{
Identification of Deregulated Transcription Factors Involved in Specific Bladder Cancer Subtypes
}

\author{
Magali Champion ${ }^{1}$, Julien Chiquet ${ }^{2}$, Pierre Neuvial ${ }^{3}$, Mohamed Elati ${ }^{4}$, \\ François Radvanyi ${ }^{5}$, and Etienne Birmelé ${ }^{1}$ \\ 1 Laboratoire MAP5, UMR8145 CNRS, Université Paris Descartes, Paris, France \\ magali.champion@parisdescartes.fr \\ 2 UMR MIA-Paris, AgroParisTech, INRA, Université Paris-Saclay, Paris, France \\ 3 Institut de Mathématiques de Toulouse, UMR 5219, Université de Toulouse, CNRS, France \\ 4 Université de Lille, CNRS, Inserm, UMR9020 - UMR-S 1277 - Canther, Lille, France \\ 5 Institut Curie, PSL Research University, CNRS, UMR144, Paris, France
}

\begin{abstract}
Comparison between tumoral and healthy cells may reveal abnormal regulation behaviors between a transcription factor and the genes it regulates, without exhibiting differential expression of the former genes. We propose a methodology for the identification of transcription factors involved in the deregulation of genes in tumoral cells. This strategy is based on the inference of a reference gene regulatory network that connects transcription factors to their downstream targets using gene expression data. Gene expression levels in tumor samples are then carefully compared to this reference network to detect deregulated target genes. A linear model is finally used to measure the ability of each transcription factor to explain these deregulations. We assess the performance of our method by numerical experiments on a public bladder cancer data set derived from the Cancer Genome Atlas project. We identify genes known for their implication in the development of specific bladder cancer subtypes as well as new potential biomarkers.
\end{abstract}

\section{Introduction}

Today, after decades of intensive research, cancer is still one of the most deadly diseases worldwide, killing millions of people every year. Cancer is mainly caused by somatic mutations that affect critical genes and pathways. These mutations are triggered by both intrinsic (like replication errors) and environmental factors (e.g. obesity, smoking, alcohol, lifestyle,...) often promoted by certain genetic configurations. In the last two decades, large-scale projects, such as the Cancer Genome Atlas project (TCGA), which has produced massive amounts of multi-omics data, have launched to improve our understanding of cancers [33]. In this context, developing statistical algorithms able to interpret these large data sets and to identify genes that are the origin of diseases and their causal pathways still remains an important challenge.

Genes are commonly affected by genomic changes in the pathogenesis of human cancer. Cancer is moreover a heterogeneous disease, with affected gene sets that may be highly different depending on subtypes, and thus requires different treatments of patients. Specific analyses of 
subtypes have for example revealed significant differences between breast cancer subgroups [13] but also pancancer similarities between breast and bladder cancer subgroups [17].

Using transcriptional data allows to look beyond DNA, that is to study abnormalities in terms of gene expression. As a common approach, differential expression analysis, for which statistical procedures have been intensively explored, can be performed and altered genes are then differentially expressed genes [12]. This points to relevant genes but does not take into account the regulations (activation and inhibition) between genes.

The approach we consider consists in taking into account the regulation structure between genes. We particularly focus on transcription factors (TFs), for their major role played in the regulation of gene expression, which make them an attractive target for cancer therapy [30, 37]. Regulation processes between TFs and their targets are usually represented by Gene Regulatory Networks (GRNs). In the last few years, many different methods have been proposed to infer GRNs from collections of gene expression data. In a discrete framework, gene expression can be discretized depending on their status (under/over-expressed or normal) and truth tables provide the regulation structure [8]. In the continuous case, regression methods, including the popular Lasso [34] and its derivatives, have provided powerful results [19, 26].

A deregulated gene then corresponds to a gene whose expression does not correspond to the expression level expected from its regulators expression. It is different from the notion of differential expression since a loss of regulation between a target gene and one of its regulating TFs implies a loss of correlation between them but not necessarily differential expression. Conversely, a TF can be differentially expressed and one of its targets not, precisely because it is deregulated.

To discover deregulated genes, a first possibility is to infer one network per condition and to compare them. Statistical difficulties due to the noisy nature of transcriptomic data and the large number of features compared to the sample size can be taken into account by inferring the networks jointly and penalizing the differences between them [3]. A second possible approach is to assess the adequacy of gene expression in tumoral cells to a reference GRN, in order to exhibit the most striking discrepancies, i.e. the regulations which are not fulfilled by the data [22, 24]. Such methods however focus on checking the validity of the network rather than highlighting genes with an abnormal behavior. Finally, analyses may be conducted at the pathway level rather than the gene level $[11,14]$. They are then not network-wide in the sense that each gene has a deregulation score by pathway it belongs to and pathways are treated independently. Moreover, as the pathways are extracted from curated databases, the regulations taken into account are not tissue-specific.

Here, we propose a statistical deregulation model that uses gene expression data to identify deregulated TFs involved in specific subtypes of cancer. This paper is organized as follows: in Section 1, we present the 3-steps method we developed and our validation procedure. In Section 2 , we illustrate its interest on the TCGA bladder cancer data set. We show that it can be used complementary to differential expression analysis to point to potential biomarkers of cancers.

\section{Methods}

\subsection{Overview of the Procedure}

Our approach for the identification of deregulated transcription factors (TFs) involved in cancers is based on a 3-steps strategy that $(i)$ creates a reference gene regulatory network (GRN), which represents regulations between groups of co-expressed TFs and target genes using a reference data set (Step 1), (ii) computes a deregulation score for each target gene in each 
tumor sample by comparing their behavior with the reference GRN (Step 2), (iii) identifies the most significant TFs involved in the deregulation of the target genes in each sample from specific cancer subtypes (Step 3). These steps are presented in Figure 1 and described in detail in the next sections.

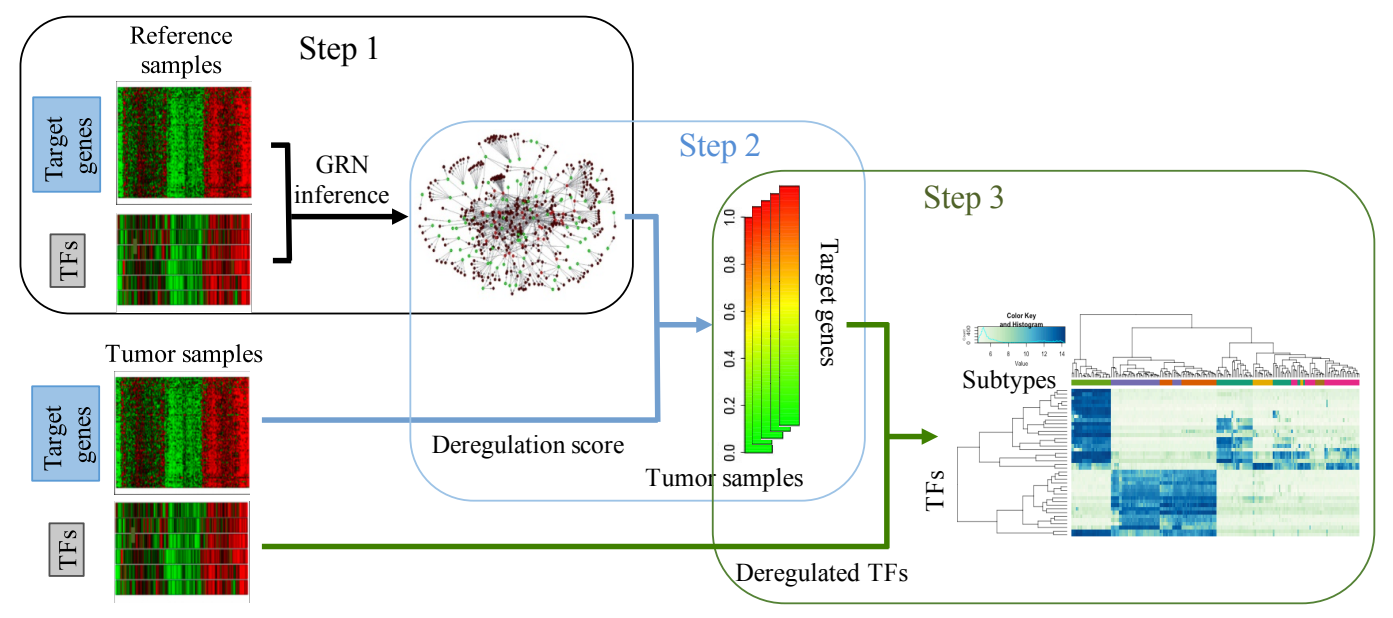

Figure 1: Workflow of the proposed 3-steps algorithm for identifying TFs involved in specific cancer subtypes.

\subsection{Step 1: Inferring a Gene Regulatory Network}

Step 1 of the algorithm consists in inferring a GRN that connects TFs to their downstream targets. Among the large number of existing methods, we choose hLICORN, available in the CoRegNet R-package [31]. This algorithm is based on a hybrid version of the LICORN model [18], in which groups of co-regulated TFs act together to regulate the expression of their targets (Figure 2). More precisely, LICORN uses heuristic techniques to identify co-activator and coinhibitor sets from discretized gene expression matrices and locally associates each target gene to pairs of co-activators and co-inhibitors that significantly explain its discretized expression. The hybrid variation of LICORN then ranks the local candidate networks according to how well they predict the target gene expression, through a linear regression, and selects the GRN that minimizes the prediction error. This selection step limits the effects of overfitting, induced by the model complexity, especially the large number of features (genes) as compared to the sample size [16]. In this work, we slightly enrich the LICORN model by creating a copy of each $\mathrm{TF}$ in the target layer to allow regulations between TFs.

To construct a specific GRN, note that one may prefer using another inference method [4] or a pre-existing regulatory network, which can be loaded from the RegNetwork database [27]. Here, we focus on hLICORN since the induced model is particularly suitable for the rest of our analysis. In addition, it was shown to provide powerful results for cooperative regulation detection, especially on cancer data set $[18,31]$. 


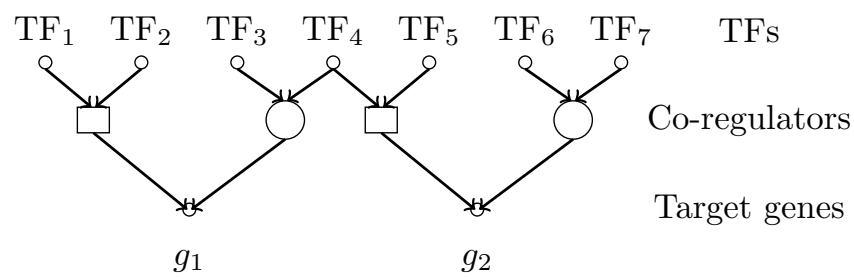

Figure 2: Example of LICORN graph involving 7 TFs and 2 target genes. TFs are gathered into groups of co-expressed genes that co-regulate (square for co-activators, circle for co-inhibitors) each target gene.

\subsection{Step 2: Computing a Deregulation Score}

Step 2 of the algorithm aims at identifying deregulated target genes by carefully comparing their expression across all tumor samples with the reference GRN inferred in Step 1. For this purpose, we use the method described in [20], which assumes that all genes from a hLICORN model are allowed to be deregulated, i.e. not to respond to their regulators as expected.

More precisely, according to the hLICORN model, each gene $g$ is connected with a set of coregulated TFs split into a group of co-activators $\mathcal{A}$ and co-inhibitors $\mathcal{I}$. A binary deregulation variable $D_{g}$, assumed to be non-zero with probability $Y$, is then introduced to compare the true status $\mathcal{S}_{g}$ (under/over-expressed or normal) of each target gene in each tumor sample with its expected value $\mathcal{S}_{g}^{*}$, resulting from a truth Table (see Figure $3(\mathrm{~b})$ ) and the inferred GRN. To avoid discretization of the data, the status of all genes are considered as hidden variables. The model is described in Figure 3 (a).

As the number of hidden variables grows exponentially with the number of genes, the likelihood of the model rapidly becomes intractable. The unknown parameters, including the deregulation score $Y$, are thus estimated using a dedicated EM-algorithm (see [20] for more details). Note that the deregulation score $Y$ does not capture information about differentially expressed genes but genes whose expression does not correspond to the level expected from its regulator expression.

\subsection{Step 3: Identifying Deregulated TFs}

Step 3 consists in identifying TFs that cause deregulations of target genes. Our approach is based on linear regression models, in which we try to explain the deregulation score of all target genes in one sample (Step 2) using their co-regulator TFs as explanatory variables (Step 1). Assume that we have $q$ TFs and $p$ target genes. Denote by $Y_{i j}$ the deregulation score of target gene $i(1 \leq i \leq p)$ in sample $j(1 \leq j \leq n)$ and $G:=\left(G_{i \ell}\right)_{1 \leq i \leq p, 1 \leq \ell \leq q}$ the GRN adjacency matrix, whose non-zero elements encode the structure (edges) of the graph. We then cast our model as follows:

$$
\forall j \in \llbracket 1, n \rrbracket, \forall i \in \llbracket 1, p \rrbracket, Y_{i j}=G_{i \ell} \cdot B_{\ell j}+\varepsilon_{i j},
$$

or, in a matrix form, $Y=G \cdot B+\varepsilon$, where each element $B_{\ell j}$ of matrix $B$, to estimate, measures the deregulation importance of TF $\ell$ in sample $j$ and $\varepsilon$ stands for the presence of noise.

Solving the $B$-estimation problem (1) can be viewed as a classical multi-task linear learning problem, in which the number of observations is the number of target genes $p$, the number of linear tasks is $n$ and the number of variables $q$. To estimate $B$, we use a constrained least 


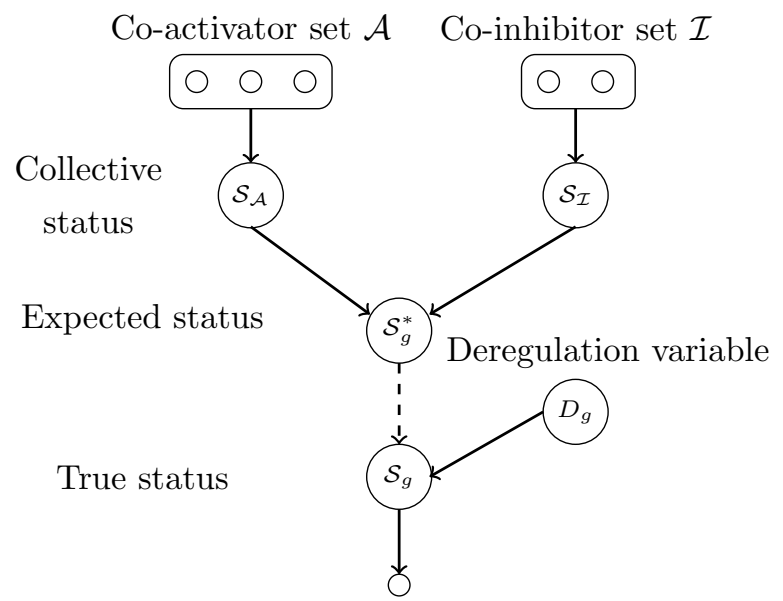

\begin{tabular}{cccc}
\hline \multirow{2}{*}{$\begin{array}{c}\text { Inhibitor } \\
\text { collective status }\end{array}$} & \multicolumn{3}{c}{$\begin{array}{c}\text { Activator } \\
\text { collective status }\end{array}$} \\
\cline { 2 - 4 } & - & 0 & + \\
\hline- & 0 & + & + \\
0 & - & 0 & + \\
+ & - & - & - \\
\hline
\end{tabular}

(b) Truth table.

$X_{g}$ expression of gene $g$

(a) Deregulation model.

Figure 3: (a) The deregulation model [20] used to compute a deregulation score for each target gene in each sample: each gene $g$ is associated to a hidden status $\mathcal{S}_{g}$ (under, over-expressed or normal). Target genes are allowed to be deregulated, i.e. not follow their co-regulator rules (Truth table (b)). The binary variable $D_{g}$ indicates whether the corresponding target gene $g$ is deregulated $\left(D_{g}=1\right)$ or not $\left(D_{g}=0\right)$. The deregulation score $Y$ of gene $g$ in sample $j$ is then the probability, given the observation, that $D_{g}=1$ in sample $j$. (b) LICORN truth table, which gives the expected status of a target gene according to the collective status of its co-activators and co-inhibitors. Collective status are set by default to 0 except if and only if all of its elements share the same status. This table is derived from biological experiments [18].

squares estimation procedure. As we only expect to find TFs positively causing the deregulation of their targets in each sample, we consider the induced constrained optimization problem:

$$
\begin{aligned}
\forall j \in \llbracket 1, n \rrbracket, \quad \hat{B}_{\cdot j} & :=\underset{\beta \in \mathbb{R}^{q}}{\operatorname{argmin}}\left\|Y_{\cdot j}-G \beta\right\|_{2}^{2}, \\
& \text { s.t } \quad \forall \ell \in \llbracket 1, q \rrbracket, 0 \leq \beta_{\ell} \leq 1
\end{aligned}
$$

where $\|\cdot\|_{2}^{2}$ stands for the euclidian norm. The closer $\hat{B}_{\ell j}$ is to 1 , the more important the role of TF $\ell$ in the deregulation of its targets in sample $j$. To solve Eq. (2), we use the limSolve R-package.

\subsection{Correcting Expression Data}

Gene expression is commonly affected by copy number alterations (CNA) [1]. Step 2 of our procedure is particularly sensitive to CNA, associating high deregulation scores to amplified or deleted target genes [20]. Indeed, the number of copies of a gene can strongly influence its expression, independently from its regulators expression, making some regulations wrongly deregulated.

To remove CNA effects on gene expression and improve the rest of our analysis, we preprocess target genes expression data beforehand as proposed in [15]. Gene expression is considered as 
linearly modified by CNA through the linear regression model:

$$
X_{i j}=\alpha_{0}+\alpha_{1} \mathrm{CNA}_{i j}+\varepsilon_{i j}
$$

where $X_{i j}$ is the expression of gene $j$ in sample $i$ and $\mathrm{CNA}_{i j}$ its associated copy number. Let $\hat{\alpha}_{0}$ and $\hat{\alpha}_{1}$ be the estimated solutions of Eq. (3), the corrected expression is then given by:

$$
\tilde{X}_{i j}=X_{i j}-\hat{\alpha}_{0}-\hat{\alpha}_{1} \mathrm{CNA}_{i j}
$$

\section{Results and Discussion}

\subsection{The Bladder Cancer Data Set}

We apply our method on bladder cancer data, produced in the framework of the Cancer Genome Atlas (TCGA) project and available at the Genomic Data Commons Data Portal (https: //portal.gdc.cancer.gov/). These data include a set of 401 bladder cancer samples with gene expression and copy number for a total number of 15,430 genes, split into 2,020 TFs and 13,410 targets. Gene expression data were produced using RNA-sequencing on bladder cancer tissues. Preprocessing is done by log-transformation and quantile-normalization of the arrays. Missing values are estimated using nearest neighbor averaging [36]. TCGA samples are analyzed in batches and significant batch effects are observed based on a one-way analysis of variance in most data modes. We apply Combat [23] to adjust for these effects. Genes are finally filtered based on their variability: among them, we only keep the $75 \%$ most varying genes.

Based on RNA-seq data analysis from the TCGA data portal, samples are split into five subtypes: basal-squamous (BaSq), luminal (Lum), luminal-infiltrated (LumI), luminal-papillary

\begin{tabular}{|c|c|c|c|c|c|}
\hline Subtypes & BaSq & Lum & LumI & LumP & $\mathrm{NE}$ \\
\hline Samples & 131 & 44 & 74 & 134 & 18 \\
\hline
\end{tabular}
(LumP) and neuronal (NE) with different characteristics [10] (Table 1).

Table 1: Molecular subtypes distribution of the 401 bladder cancer samples [10] .

\subsection{Description of the Procedure Results}

GRN network. To validate our method, we have to provide a tissue-specific reference GRN (Step 1), which is computed given a first set of reference samples. In many cancers, the pure normal tissue of origin is not available. Here, we work with the five different subtypes of the TCGA data set presented in Section 3.1. Using samples from one subtype as test cases and the rest as reference, we infer five different GRNs. Each of them reflect averaged relationships between genes for patients who are not part of one specific subtype. Due to the very-high heterogeneity of cancers, especially of bladder cancers [25, 35], we think that our method will still point to relevant deregulations of specific subtypes.

After calibrating the internal parameters of the hLICORN agorithm, the GRNs we infer are made of an averaged total number of 28,246 edges connecting 586 TFs to 3,432 of their targets. These networks are relatively sparse, each of the target genes being associated with an averaged number of 8 TFs. 
Deregulation scores. We then run five times the EM procedure (Step 2) on the five subsets of the gene expression data matrix to compute a deregulation score of each target gene in each sample of each subtype. From now on, all samples are treated individually, the results reflecting how genes behaved in each sample of one subtype in comparison to reference samples from all other subtypes.

To check the effect of the copy number correction we apply at the beginning of our procedure, we compare the distribution of the deregulation scores across copy number states. To this aim, we use the TCGA CNA thresholded data set, which associates to each gene-sample pair a copy number state of "0" for the diploid state (two copies), "1" for a copy number gain, "-1" for a copy number loss, "2" for an amplification and "-2" for a deletion. We then test for significant differences between the diploid state and the altered states $(-2,-1,1,2)$ using Student tests. Results in terms of p-values, which are corrected for multiple hypothesis testing using the FDR [2], are presented in Table 2. With corrected p-values ranging from 0.10 to 1 , deregulation scores are no longer associated with CNA.

\begin{tabular}{|c|c|c|c|c|c|c|c|c|c|c|c|c|c|c|c|c|c|c|c|}
\hline \multicolumn{20}{|c|}{ Subtypes } \\
\hline \multicolumn{4}{|c|}{$\mathrm{BaSq}$} & \multicolumn{4}{|c|}{ Lum } & \multicolumn{4}{|c|}{ LumI } & \multicolumn{4}{|c|}{ LumP } & \multicolumn{4}{|c|}{$\mathrm{NE}$} \\
\hline-2 & -1 & 1 & 2 & -2 & -1 & 1 & 2 & -2 & -1 & 1 & 2 & -2 & -1 & 1 & 2 & -2 & -1 & 1 & 2 \\
\hline 1 & 0.81 & 0.28 & 1 & 1 & 1 & 1 & 1 & 1 & 0.25 & 0.10 & 0.60 & 1 & 1 & 1 & 1 & 1 & 1 & 1 & 1 \\
\hline
\end{tabular}

Table 2: Corrected p-values for Student tests when comparing the distribution of the deregulation scores between the diploid state $(0)$ and each altered state $(-2,-1,1,2)$ for each subtype.

Deregulated TFs. We finally apply Step 3 of our method to identify TFs involved in the deregulation scores of the target genes, that is having a non-zero coefficient in $\hat{B}$, as given in Eq. (1). We then rank the TFs according to their number of non-zero coefficients across all samples belonging to each specific subtype. Results are presented in Table 3.

\begin{tabular}{|c|c|c|c|c|c|c|c|c|c|}
\hline \multicolumn{10}{|c|}{ Subtypes } \\
\hline \multicolumn{2}{|c|}{$\mathrm{BaSq}$} & \multicolumn{2}{|c|}{ Lum } & \multicolumn{2}{|c|}{ LumI } & \multicolumn{2}{|c|}{ LumP } & \multicolumn{2}{|l|}{$\mathrm{NE}$} \\
\hline $\mathrm{TF}$ & $\% \hat{B}$ & $\mathrm{TF}$ & $\% \hat{B}$ & $\mathrm{TF}$ & $\% \hat{B}$ & $\mathrm{TF}$ & $\% \hat{B}$ & $\mathrm{TF}$ & $\% \hat{B}$ \\
\hline SPOCD1 & $92 \%$ & ZNF268 & $91 \%$ & TSHZ1 & $88 \%$ & RARB & $84 \%$ & FAIM3 & $89 \%$ \\
\hline ZNF382 & $86 \%$ & HES2 & $80 \%$ & ZNF354B & $88 \%$ & RFX5 & $84 \%$ & SMARCA2 & $83 \%$ \\
\hline RCOR2 & $86 \%$ & TBX2 & $80 \%$ & $\mathrm{AR}$ & $85 \%$ & CBFA2T3 & $83 \%$ & RARB & $78 \%$ \\
\hline ATM & $83 \%$ & PRDM8 & $75 \%$ & HES2 & $82 \%$ & TBX18 & $81 \%$ & ZNF235 & $78 \%$ \\
\hline HABP4 & $83 \%$ & TSHZ1 & $75 \%$ & HTATIP2 & $81 \%$ & TBX3 & $79 \%$ & TBX2 & $72 \%$ \\
\hline IRX3 & $82 \%$ & ZNF354C & $73 \%$ & MAFG & $80 \%$ & PTRF & $79 \%$ & STAT3 & $72 \%$ \\
\hline IFI16 & $79 \%$ & RARB & $70 \%$ & ENO1 & $80 \%$ & TBX2 & $70 \%$ & HIF1A & $72 \%$ \\
\hline TEAD2 & $79 \%$ & KLF13 & $70 \%$ & TBX2 & $74 \%$ & PPARG & $76 \%$ & THRA & $72 \%$ \\
\hline NOTCH4 & $79 \%$ & SCML2 & $68 \%$ & ZNF563 & $74 \%$ & NCOR2 & $75 \%$ & PIR & $67 \%$ \\
\hline SNAI2 & $79 \%$ & SNAI3 & $68 \%$ & IRX3 & $72 \%$ & ZFP2 & $75 \%$ & FOSL1 & $67 \%$ \\
\hline
\end{tabular}

Table 3: List of the 10 most important TFs for explaining the deregulation scores of their targets and number of non-zero coefficients in $\hat{B}$ (in \%) across all samples from each subtype. 


\subsection{Discussion}

Top TFs include biomarkers of bladder cancer. Among TFs of Table 3, we retrieve characteristic genes of bladder cancer subtypes. For instance, SNAI2, which is deregulated across $79 \%$ of the BaSq samples, is particularly well-known for its implication in EMT pathways for cancer patients [6] and its capacity to discriminate between basal and luminal subgroups [29]. The presence of NOTCH4 in BaSq samples is particularly interesting as it is part of the NOTCH pathway, whose inactivation tends to promote bladder cancer progression [28]. Research works also focus on its implication on the basal subgroup [21]. Similarly, TBX2, involved in all three luminal subtypes is an indicator of luminal cancers [7]. We can finally emphasize the presence of PPARG in LumP, a TF whose high level of expression is used to describe luminal subtypes and which has been shown to have a protumorigenic role in these subtypes [5, 9].

Deregulation is complementary to differential gene expression analysis Differential gene expression analysis consists in performing statistical analysis to discover quantitative changes in terms of expression levels between groups. It is frequently used in cancer research to identify genes with important changes between tumor and normal samples, called differentially expressed genes (DEGs) [32].

We perform differential gene expression analysis using the R-package limma [32] on all samples from each subtype when comparing to samples from all other subtypes. We then verify whether the identified DEGs are different from the deregulated TFs derived from our method (Figure 4). To this aim, we use the following thresholds: a gene is called DEG for p-values smaller than 0.01 whereas it is deregulated for a subtype as soon as it is deregulated $(\hat{B} \neq 0)$ for more than the $50 \%$ of the subtype samples. This threshold is purely arbitrary but is not crucial, as the results remain almost the same with slight changes. As shown in Figure 4, except for BaSq and LumP subtypes, more than the $70 \%$ of the identified deregulated TFs are not differentially expressed, which means that our procedure does not only point to DEGs.
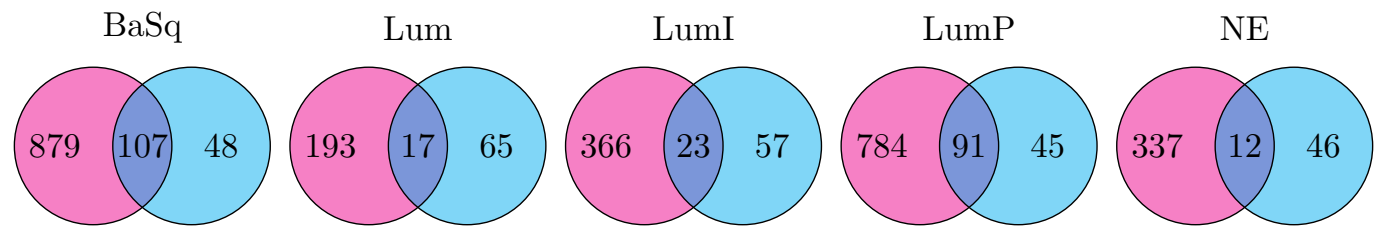

Figure 4: Venn Diagrams representing the number of DEGs (in pink), the number of deregulated TFs identified by our method (in blue) and their intersection.

\section{Conclusion}

With the aim of understanding the deregulation processes in tumoral cells, we develop a 3-steps strategy that measures the influence of TFs in the deregulation of genes in tumor samples. A list of TFs characterizing given subtypes can then be established. Even if a biological experimental validation should be done in future work, it seems that it can be used complementary to differential gene expression analysis to point to potential biomarkers of cancers.

An open question, which has also to be tackled, is to determine in which extend the information carried by mutations can explain the deregulations. Mutation data are particularly 
hard to explore in this context due to various reasons : first of all, mutations do not necessarily affect gene expression. Secondly, in cancers, besides the most significant mutated genes, many sequencing projects have shown that genes are mutated in less than $5 \%$ of the samples.

In this work, among the identified TFs of Table 3, ATM is highly deregulated (83\%) and mutated (15\%) for BaSq samples. Mutations of ATM have been recently shown to be associated with shorter survival in urothelial cancers [38]. As a preliminary result, we observe that $95 \%$ of the mutated BaSq samples, i.e 18 of the 19 samples, corresponds to non-zero $\hat{B}$ coefficients. However, the number of samples is still too small to positively conclude for a significant association. Supplementary works need to be done to go further.

Availability of supporting data The algorithm (written in $\mathrm{R}$ ) and data described in this article are available on GitHub at https://github.com/magalichampion/LIONS_project.

\section{References}

[1] P. Aldred, E. Hollox, and J. Armour. Copy number polymorphism and expression level variation of the human alpha-defensin genes DEFA1 and DEFA3. Hum Mol Genet, 14(14):2045-2052, 2005.

[2] Y. Benjamini and Y. Hochberg. Controlling the false discovery rate: a practical and powerful approach to multiple testing. J R Stat Soc Ser B Methodol, 57(1):289-300, 1995.

[3] J. Chiquet, Y. Grandvalet, and C. Ambroise. Inferring multiple graphical structures. Stat Comput, 21(4):537-553, 2011.

[4] J. Chiquet, Y. Grandvalet, and C. Charbonnier. Sparsity in sign-coherent groups of variables via the cooperative-lasso. Ann Appl Stat, 6:795-830, 2012.

[5] W. Choi et al. Identification of basal and luminal subtypes of muscle-invasive bladder cancer with different sensitivities to frontline chemotherapy. Cancer cell, 25(2):152-165, 2014.

[6] C. Cobaleda, M. Pérez-Caro, C. Vicente-Dueñas, and I. Sànchez-Garcia. Function of the zinc-finger transcription factor SNAI2 in cancer and development. Annu Rev Genet, 41:41-61, 2007.

[7] D. Dhawanet al. Comparative gene expression analyses identify luminal and basal subtypes of canine invasive urothelial carcinoma that mimic patterns in human invasive bladder cancer. PLoS One, 10(9):e0136688, 2015.

[8] M. Elati and C. Rouveirol. Unsepervised learning for Gene Regulation Network Inference from Expression Data: a review. John Wiley and Sons, Inc., Hoboken, NJ, 2011.

[9] A. Biton et al. Independent component analysis uncovers the landscape of the bladder tumor transcriptome and reveals insights into luminal and basal subtypes. Cell Rep., 9(4):1235-45, 2014.

[10] A. G. Robertson et al. Comprehensive molecular characterization of muscle-invasive bladder cancer. Cell, 171(3):540-556, 2017.

[11] A.L. Tarca et al. A novel signaling pathway impact analysis. Bioinformatics, 25(1):75-82, 2009.

[12] B. Kaczkowski et al. Transcriptome analysis of recurrently deregulated genes across multiple cancers identifies new pan-cancer biomarkers. Cancer Research, 76(2):216-226, 2016.

[13] B.D. Lehman et al. Identification of human triple-negative breast cancer subtypes and preclinical models for selection of targeted therapies. J Clin Invest, 121(7):2750-2767, 2011.

[14] C.J. Vaske et al. Inference of patient-specific pathway activities from multi-dimensional cancer genomics data using paradigm. Bioinformatics, 26:i237-i245, 2010.

[15] E.I. Delatola et al. Segcorr: a statistical procedure for the detection of genomic regions of correlated expression. BMC Bioinformatics, 18(1):333, 2017.

[16] I. Chebil et al. Hybrid method inference for the construction of cooperative regulatory network in human. IEEE Trans Nanobioscience, 13(2):97-103, 2014. 
[17] J.S. Damrauer et al. Intrinsic subtypes of high-grade bladder cancer reflect the hallmarks of breast cancer biology. Proc Natl Acad Sci USA, 111(8):3110-3115, 2014.

[18] M. Elati et al. Licorn: learning cooperative regulation networks from gene expression data. Bioinformatics, 23(18):2407-14, 2007.

[19] M. Vignes et al. Gene regulatory network reconstruction using bayesian networks, the dantzig selector, the lasso and their meta-analysis. PloS one, 6(12):e29165, 2011.

[20] T. Picchetti et al. A model for gene deregulation detection using expression data. BMC Systems Biology, 9:S6, 2015.

[21] A. Greife et al. Canonical notch signalling is inactive in urothelial carcinoma. BMC Cancer, 14:628, 2014.

[22] C. Guziolowski, A. Bourde, F. Moreews, and A. Siegel. Bioquali cytoscape plugin: analysing the global consistency of regulatory networks. BMC Genomics, 10(1):244, 2009.

[23] W.E. Johnson, C. Li, and A. Rabinovic. Adjusting batch effects in microarray expression data using empirical bayes methods. Biostatistics, 8:118-127, 2007.

[24] G. Karlebach and R. Shamir. Constructing logical models of gene regulatory networks by integrating transcription factor-DNA interactions with expression data: An entropy-based approach. J Comput. Biol, 19(1):30-41, 2012.

[25] M.A. Knowles and C.D. Hurst. Molecular biology of bladder cancer: new insights into pathogenesis and clinical diversity. Nat Rev Cancer, 15:25-41, 2014.

[26] B. Liu, A. de la Fuente, and I. Hoeschele. Gene network inference via structural equation modeling in genetical genomics experiments. Genetics, 178(3):1763-1776, 2008.

[27] Z. Liu, W. Canglin, H. Miao, and H. Wu. Regnetwork: an integrating database of transcriptional and post-transcriptional regulatory networks in human and mouse. Database (Oxford), 2015, 2015.

[28] A. Maraver et al. Notch pathway inactivation promotes bladder cancer progression. J Clin Invest, 125(2):824-830, 2015.

[29] D.S. Mistry, Y. Chen, Y. Wang, and G.L. Sen. SNAI2 controls the undifferentiated state of human epidermal progenitor cells. Stem Cells, 32(12):3209-3218, 2014.

[30] D.W. Nebert. Transcription factors and cancer: an overview. Toxicology, 181-182:131-141, 2002.

[31] R. Nicolle, F. Radvanyi, and M. Elati. Coregnet: reconstruction and integrated analysis of coregulatory networks. Bioinformatics, 31(18):3066-3068, 2015.

[32] M. E. Ritchie et al. limma powers differential expression analyses for RNA-sequencing and microarray studies. Nucleid Acids Res, 43(7):e47, 2015.

[33] The Cancer Genome Atlas. Comprehensive molecular characterization of urothelial bladder carcinoma. Nature, 507(7492):315-322, 2014.

[34] R. Tibshirani. Regression shrinkage and selection via the lasso. J R Statist Soc Ser B, 58(1):267288, 1996.

[35] F.S. Togneri et al. Genomic complexity of urothelial bladder cancer revealed in urinary cfDNA. Eur J Human Genet, 24:1167-1174, 2016.

[36] O. Troyanskaya et al. Missing value estimation methods for DNA microarrays. Bioinformatics, 17(6):520-525, 2001.

[37] J.E. Yeh, P.A. Toniolo, and D.A. Frank. Targeting transcription factors: promising new strategies for cancer therapy. Current Opinion in Oncology, 25(6):652-658, 2013.

[38] M. Yin et al. ATM/RB1 mutations predict shorter overall survival in urothelial cancer. Oncotarget, 9(24):16891-16898, 2018. 